\title{
Therapeutic Nutrition for Children with Severe Acute Malnutrition: Summary of African Experience
}

\author{
ANdré Briend ANd STeve Collins* \\ From the Department of International Health, University of Tampere, Finland; and *Valid International Ltd, 35, Leopold Street, \\ Oxford, OX4 $1 \mathrm{TW}, \mathrm{UK}$. \\ Correspondence to: Dr Steve Collins, Valid International Ltd, 35, Leopold Street, Oxford, OX4 1 TW, UK. \\ steve@validinternational.org.uk
}

Across Africa, Severe Acute Malnutrition (SAM) affects approximately $3 \%$ of children under five at any time and is associated with several hundred thousand child deaths each year. Since the 1950s, efforts to treat these children as inpatients in hospitals or clinics have failed to lower mortality rates and have achieved very poor coverage. During the past 10 years new community-based management approaches treating over $85 \%$ of SAM cases solely as outpatients using nutrient dense, lipid-based Ready to Use Therapeutic Foods have dramatically reduced mortality and increased coverage rates. In 2005, this new model was endorsed by the UN under the name Community-based Management of Acute Malnutrition (CMAM) and has now been adopted by over 25 National governments and all major relief agencies. By 2009 , approximately 1 million cases of SAM were being treated annually, with programs expanding by approximately $30 \%$ year on year.

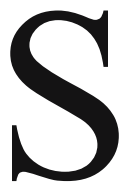
evere Acute Malnutrition (SAM) is common in sub-Saharan Africa, with approximately $3 \%$ of children under five affected at any one time, it is also associated with several hundred thousand child deaths each year. Between 1950 and 2000, efforts to treat these children as inpatients in district hospitals or clinics failed to address the problem; mortality rates amongst those undergoing treatment remained extremely high at 20-30\% and unchanged from those seen in the $1950 \mathrm{~s}(1)$, and only a tiny proportion of those in need were able to access care(2). Over the same period, every time a major crisis emerged in Africa, relief agencies established inpatient feeding centres to treat the large number of children requiring care. During the $80 \mathrm{~s}$ and $90 \mathrm{~s}$, research into rehabilitation diets rapidly allowed these centres to lower mortality rates amongst those treated to $<10 \%$; however, coverage rates remained very low and the centres themselves were costly to implement and undermined the existing infrastructure. In response to these problems, agencies working in collaboration with National governments in Malawi and Ethiopia, developed simple effective community-based treatment models that could be implemented rapidly on large numbers of SAM children with minimum resources(3). Major progress has been achieved over the past ten years in refining this new model and extending its implementation. By 2005, when the model was endorsed by the UN(4), mortality rates had been reduced to $<5 \%$ and coverage rates increased to $>50 \%(5)$. By 2009, all major relief agencies and 25 National governments had formally adopted this new model and approximately 1 million cases of SAM were treated annually, with programs expanding by approximately $30 \%$ every year.

\section{The InPatient Treatment ApProACH}

The initial efforts to treat large numbers of children with severe acute malnutrition emerged during the Ethiopia famine of the mid-70s(6). Children were then admitted to large inpatient feeding centres based on their arm circumference for height or for age, and then treated with a mixture of dried skimmed milk, oil, and sugar, diluted into clean water. At that time, it was already acknowledged that SAM children needed additional potassium and 
magnesium, and these minerals were added to the diet. Other minerals and vitamins were given directly.

Over the years, changes were made to this protocol; a standard mineral mix was developed in the mid-90s(7) based on research carried out in Jamaica to promote not only weight gain, but also recovery of different body functions. Ready-made recovery diets, based on the same basic formula with added improved vitamin and mineral mix became available in the mid-90s and rapidly became widely used to minimize risk of errors in the preparation of feeds. A starter diet, F-75, with lower protein and sodium content was introduced around the same time. With these new dietary protocols and relatively intensive medical care, recovery rates improved often exceeding $75 \%$. Mortality rates were reduced to below $10 \%$. From a clinical perspective, these protocols were considered as rather successful.

However, at the same time, the limitations of the inpatient model, became clear. Although successful in terms of recovery of treated children, it failed to tackle SAM as a public health problem. Demand on staff and infrastructure were so large that the model was not sustainable outside emergency situations where these protocols were implemented by international, well funded organisations. Also, in terms of coverage, i.e. the number of children effectively treated compared to the total number of children with SAM, remained very low. This was especially the case in open situations amongst dispersed communities living in chronic poverty where often specialized inpatient feeding centers were not accessible. In their absence, children had to be referred to overcrowded pediatric or general wards ill-equipped to give adequate care to malnourished children. Having the mother staying with the child for several weeks was also a major problem when they were engaged in agriculture or other life sustaining activities.

\section{Mid-90s: Development of the Community Based Management}

The limitations of the inpatient model led to innovations in the mid-90s, which progressively introduced a radically new approach, moving the treatment of SAM from inpatient hospitals and feeding centres into the community. Three key innovations underpinned this revolution in care: $(i)$ the introduction of technique to engage with communities to promote early presentation and compliance(2); (ii) handing over the identification of SAM to the community through the use of MUAC; and (iii) the development of Ready to Use Therapeutic Foods (RUTF). The overall effect of these changes was to make treatment accessible, understandable and affordable (in terms of opportunity costs to poor people). The result was that people presented for treatment early and in large numbers, at a time when their condition was still easily treatable at home.

\section{Community Engagement}

During the development of the community-based model, anthropological studies showed that most people did not understand the bio-medical model of SAM and instead sought treatment with traditional practitioner(8). This prolonged the time to presentation, thereby increasing the frequency of complications. These studies also observed that the inpatient model, which separated families and took mothers and children away from husbands, siblings and farms was unpopular; and this unpopularity further delayed the presentation and reduced the compliance.

The new community-based approach was based upon proactive engagement with communities combined with a simple outpatient treatment model that, so long as children presented for treatment early, resulted in the vast majority not requiring admission into hospitals or clinics. The community-engagement recognized that the decision making process relied upon key individuals within families and communities and once these people understood and appreciated the care provided, early presentation and case finding increased dramatically. Based around local capacity and simple measures to inform and engage with people, this model was easily implemented and resourced even in impoverished environments.

2. Decentralization of Access and the Use of MUAC for Diagnosis of SAM

Early anthropological work also identified access 
and diagnosis of SAM as major barriers to early admission and compliance(9). To address this, the community-based model adopted a system of decentralized points of access using the lowest levels of the existing public health system (health posts) to screen and admit patients. The aim was to enable the client to travel to and from the clinic and receive treatment in less than a day. To simplify the admission procedures, avoid confusion with ongoing growth monitoring activities and facilitate community-based screening by volunteer workers, MUAC was chosen as the anthropometric measure of choice(10).

The use of MUAC is easy, fast to perform on large numbers of children and is understandable to patients and staff. It also removes confusion with growth monitoring weight for age indicators. MUAC without correction for age or height was used, as early studies from Africa and Bangladesh showed that correction of MUAC for age or height did not improve the assessment of the risk of death $(11,12)$. The value of MUAC to identify children with a high risk of death may be related to the selection of younger children who have a higher risk. However, the close relationship of MUAC with muscle mass $(13,14)$ and the importance of muscle in general metabolism(15) suggest that it may have a specific physiological meaning. In any case, the use of MUAC has greatly simplified the process of case identification and admission, simplified protocols, reduced staff workloads, and reduced training needs.

\section{The Use of Ready to Use Therapeutic Food (RUTF)}

Treatment with liquid diets used since the 1970s was only possible in inpatient settings, as liquid foods, whether milk based or traditional cereal based foods, are excellent media for growth of pathogenic bacteria. As a result, if water used for preparing these feeds is not prepared or stored in perfect hygienic conditions, pathogenic bacteria grow rapidly in them and they may induce life threatening diarrhea(16). In these conditions, contaminated foods often contain more than 10 times as many pathogenic bacteria than water itself(17). To prevent this risk, liquid diets had to be used with perfectly clean water and in places where refrigeration was not possible; discarded if not consumed immediately, which meant they had to be prepared as many times as children were fed every day. In practice, this was possible only in inpatient facilities where children had to stay until their recovery was achieved, which usually took several weeks.

To avoid this problem, a simple solution was to use a Ready-to-Use Therapeutic Food (RUTF) which can be consumed directly by the child without the addition of water. In 1997, it was proposed to use a diet with a nutritional composition very close to the F-100 diet recommended by WHO for the recovery phase(18) obtained by replacing in the original recipe about half of the dried skimmed milk with peanut butter, removing all the water and increasing the amount of oil(19). The elimination of water from the RUTF recipe removes the dangers of bacteria contamination as bacteria need water to grow, making this food safe for use in the community. Acceptability and efficacy trials proved encoura$\operatorname{ging}(20,21)$, and the major constraint imposing that children should stay for several weeks in the inpatient centres was removed. Inpatient treatment of SAM became necessary only for complicated forms of SAM and only until medical complications requiring medical attention were addressed(22).

The change of the initial F-100 recipe needed to produce RUTF is minimal, and in theory it can be produced in any health facility with a clean kitchen and a mixer(23). Studies have demonstrated an equivalent efficacy of RUTF made locally in Malawi and Senegal to product imported from France using a more complex recipe $(24,25)$. RUTF is now manufactured to international quality standards in several countries across Africa. This has reduced the cost of RUTF, created some employment and upgraded food manufacturing in several countries; it also provides some demand local farm crops that through the targeted purchase from small holder farmers is providing economic benefits back to vulnerable groups(26).

\section{Conclusion}

The move to the community-based treatment of SAM has made it possible to address SAM as a public health problem treating millions of children 
across 35 countries in the past few years. This change has addressed key capacity constraints especially those of trained staff and hospital inpatient capacity, that previously limited both the numbers of children treated and the quality of treatment for those that did manage to gain access. The result is huge improvements in program coverage and greatly reduced mortality rates. Community-based management using RUTF has reduced costs and, in terms of the cost per disability adjusted life year (DALY) gained, delivers a cost effectiveness comparable to mainstream public health interventions such as vitamin A distribution(27). The local production of RUTF using local crops grown by small holder farmers affords a mechanism to link nutritional treatment with improvements in agricultural incomes that, if targetted correctly, could be a valuable tool to improve food security in selected vulnerable populations.

Contributors: Both authors collaborated on all parts of the article.

Funding: None.

Conflict of interest: None stated.

\section{REFERENCES}

1. Schofield C, Ashworth A. Why have mortality rates for severe malnutrition remained so high? Bull World Health Organ 1996; 74: 223-229.

2. Damme WV, Boelaert M. Therapeutic feeding centres for severe malnutrition. Lancet 2002; 59: 260-261.

3. Collins S, Sadler K. The outpatient treatment of severe malnutrition during humanitarian relief programmes. Lancet 2002; 360: 1824-1830.

4. Community-based management of Severe Acute Malnutrition-A Joint Statement by the World Health Organization, the World Food Programme, the United Nations Standing Committee on Nutrition and the United Nations Children's Fund. Geneva: UN Press; 2007.

5. Collins S, Dent N, Binns P, Bahwere P, Sadler K, Hallam $\mathrm{H}$, et al. Management of severe acute malnutrition in children. Lancet 2006; 368: 19922000.

6. Mason JB, Hay RW, Leresche J, Peel S, Darley S. Treatment of severe malnutrition in relief. Lancet.
$1974 ; 1: 332-335$.

7. Golden MH, Briend A. Treatment of malnutrition in refugee camps. Lancet 1993; 342: 360.

8. Gerrero, S, Khara, T. Community Participation and Mobilisation in CTC. ENN Special Supplement Series, No. 2. Oxford: Emergency Nutrition Network; 2004.

9. Guerrero,S, Myatt, M, Collins, S. Determinants of coverage in community-based therapeutic care programs: towards a joint quantitative and qualitative analysis. Disasters 2009; 34: 571-585.

10. Myatt M, Khara T, Collins S. A review of methods to detect cases of severely malnourished children in the community for their admission into communitybased therapeutic care programs. Food Nutr Bull 2006;27: S7-23.

11. Briend A, Zimicki S. Validation of arm circumference as an indicator of risk of death in one to four year old children. Nutr Res 1986; 6: 249-261.

12. Briend A, Garenne M, Maire B, Fontaine O, Dieng K. Nutritional status, age and survival: the muscle mass hypothesis. Eur J Clin Nutr 1989; 43: 715-726.

13. Trowbridge FL, Hiner CD, Robertson AD. Arm muscle indicators and creatinine excretion in children. Am J Clin Nutr 1982; 36: 691-696.

14. Brambilla P, Rolland-Cachera MF, Testolin C, Briend A, Salvatoni A, Testolin G, et al. Lean mass of children in various nutritional states. Comparison between dual-energy X-ray absorptio-metry and anthropometry. Ann N Y Acad Sci 2000; 904: 433436.

15. Wolfe RR. The underappreciated role of muscle in health and disease. Am J Clin Nutr 2006; 84: 475482.

16. Rowland MG, Barrell RA, Whitehead RG. Bacterial contamination in traditional Gambian weaning foods. Lancet 1978; 1: 136-8.

17. Black RE, Brown KH, Becker S, Alim AR, Merson MH. Contamination of weaning foods and transmission of enterotoxigenic Escherichia coli diarrhoea in children in rural Bangladesh. Trans $\mathrm{R}$ Soc Trop Med Hyg 1982; 76: 259-264.

18. World Health Organization, Management of Severe Malnutrition: A manual for physicians and other Senior Health Workers, Geneva: WHO; 1999.

19. Briend A. Treatment of Severe Malnutrition with a Therapeutic Spread. ENN Field Exchange, 1997; 3: 
15. Available at: http://fex.ennonline.net/2/ treatment.aspx. Accessed April 30, 2010.

20. Briend A, Lacsala R, Prudhon C, Mounier B, Grellety Y, Golden MH. Ready-to-use therapeutic food for treatment of marasmus. Lancet 1999; 353: 1767-1768.

21. Diopel HI, Dossou NI, Ndour MM, Briend A, Wade S. Comparison of the efficacy of a solid ready-to-use food and a liquid, milk-based diet for the rehabilitation of severely malnourished children: a randomized trial. Am J Clin Nutr 2003; 78: 302-307.

22. Collins S, Yates R. The need to update the classification of acute malnutrition. Lancet 2003; 362: 249.

23. Manary MJ. Local production and provision of ready-to-use therapeutic food (RUTF) spread for the treatment of severe childhood malnutrition. Food Nutr Bull 2006; 27: S83-89.
24. Sandige H, Ndekha MJ, Briend A, Ashorn P, Manary MJ. Home-based treatment of malnourished Malawian children with locally produced or imported ready-to-use food. J Pediatr Gastroenterol Nutr 2004; 39: 141-146.

25. Diop EI, Dossou NI, Briend A, Yaya MA, Ndour MM, Wade S. Home-based Rehabilitation for Severely Malnourished Children Using Locally Made Ready-to-use Therapeutic Food. (RTUF) Reports from the 2nd World Congress of Pediatric Gastroenterology, Hepatology and Nutrition. Paris (France), July 3-7, 2004. Medimond, Monduzzi Editore (International Proceedings) pp. 101-105.

26. Ortez, M. Field exchange, April 2010. Available at http://www.ennonline.net/pool/files/fex/fx-38final-copy.pdf . Accessed 30 April, 2010.

27. Bachmann MO. Cost effectiveness of communitybased therapeutic care for children with severe acute malnutrition in Zambia: decision tree model. Cost Eff Resour Alloc 2009; $7: 2$. 